\title{
Le sourire énigmatique de la vie
}

\section{Jean-Claude Gens}

\section{OpenEdition}

\section{Journals}

Édition électronique

URL : http://journals.openedition.org/alter/807

DOI : 10.4000/alter.807

ISSN : 2558-7927

\section{Éditeur :}

Association ALTER, Archives Husserl (CNRS-UMR 8547)

\section{Édition imprimée}

Date de publication : 1 novembre 2013

Pagination : 89-100

ISBN : 978-2-95-223749-9

ISSN : 1249-8947

Référence électronique

Jean-Claude Gens, « Le sourire énigmatique de la vie », Alter [En ligne], 21 | 2013, mis en ligne le 01 juin 2019, consulté le 07 juillet 2019. URL : http://journals.openedition.org/alter/807 ; DOI : 10.4000/ alter.807 


\section{LE SOURIRE ÉNIGMATIQUE DE LA VIE}

Jean-Claude Gens

On pourrait espérer que, plus que n'importe quelle autre, les philosophies qui se sont vues dénommées ou se sont elles-mêmes intitulées "philosophie de la vie » nous éclairent sur ce qu'il faut entendre par ce terme de "vie ». Sauf que l'unité d'une telle philosophie est problématique au regard de la diversité des figures qui l'incarnent. Sans remonter à l'époque où elle émerge, par exemple dans la pensée de Herder, puis se prolonger dans le romantisme, la vie est comme l'avance Herbert Schnädelbach ${ }^{1}$ le thème dominant de la philosophie entre 1880 et 1933: il suffit de penser à Nietzsche, Dilthey, Simmel, Bergson, Spengler, Klages, et, plus tard à Uexküll qui, en 1930, a publié une Lebenslehre (Doctrine de la vie), ou même à Michel Henry. Il est en ce sens plus facile de circonscrire négativement non tant un courant qu'une tendance ou une orientation, puisque, pour reprendre une expression de Friedrich Bollnow, nous avons ici d'abord affaire à un Kampfbegriff, un concept polémique ${ }^{2}$. Cette dimension polémique des philosophies de la vie vise, comme on sait, à la fois la prétention de saisir conceptuellement et systématiquement la concrétude du réel, c'est-à-dire sa fluidité ou sa mobilité, et la conception du sujet humain qui le réduit à un ensemble de facultés essentiellement théoriques. Comme dans la seconde Inactuelle de Nietzsche sur l'histoire, cette critique trouve souvent simultanément son prolongement naturel dans une Kulturkritik.

\footnotetext{
${ }^{1}$ Herbert Schnädelbach, Philosophie in Deutschland, 1831-1933, Francfort-sur-le-Main, Suhrkamp, 1994, p. 172.

${ }^{2}$ O. F. Bollnow, Lebensphilosophie (1958), in Schriften IV, Lebensphilosophie und Existenzphilosophie, Würzburg, Königshausen \& Neumann, 2009, p. 12 ; une traduction française de cet essai est disponible à l'adresse www.otto-friedrich.bollnow.de/doc/PhilosophiedelaVie.pdf.
} 
Cette tendance ou cette orientation a, de ce fait, souvent eu mauvaise presse dans la mesure où on l'a suspecté de conduire à une naturalisation de la réalité culturelle et historique, ou de reconduire celle-ci à l'intimité de vécus, ce qui ferait de cette philosophie une expression du subjectivisme moderne. Plus généralement, on y a vu l'expression d'un irrationalisme, ce qui permettait évidemment de l'écarter à moindres frais. Je pense ici aussi bien à la critique de Heinrich Rickert dont la Philosophie des Lebens de 1920 vise une philosophie "à la mode», qu'à celle de Schnädelbach. Ce dernier invite à penser la philosophie de la vie comme une métaphysique, puisque le statut de la vie est celui d'un principe, et plus précisément comme une « métaphysique de l'irrationnel $»^{3}$ puisqu'un tel principe est irrationnel. Quel que puisse être son bien-fondé, il est néanmoins permis de douter de la pertinence de ce genre de qualification d'abord au regard de son ambiguïté et de sa pauvreté. Elle est ambiguë étant donné la diversité des acceptions du terme de métaphysique, dont témoigne le fait que les critiques nietzschéenne et diltheyenne qualifient de leur côté de métaphysique la pensée contre laquelle elles s'élaborent. Elle est pauvre dans la mesure où elle s'inscrit dans le cadre d'une pensée binaire qui réduit la diversité des pensées philosophiques à une simple alternative. Par ailleurs le qualificatif d'irrationnel n'est jamais que celui qui est invoqué par un certain type de philosophie pour rejeter celles qui s'en démarquent. L'idée que la vie serait le «principe » des philosophies de la vie est en outre plus problématique qu'il ne semble à première vue dans la mesure où certaines philosophies de la vie se caractérisent précisément par la thèse selon laquelle il nous est impossible de nous assurer d'un principe ou d'un fondement au sens cartésien de ce terme, les philosophies par exemple de Nietzsche ou de Dilthey. Celles-ci se distinguent au contraire par leur caractère antifondationnel et voient dans la vie une réalité essentiellement insondable (unergründlich), ou énigmatique (rätselhaft) ou encore, comme l'écrit parfois Dilthey, un mystère (Geheimnis). Si ce thème de l'insondabilité de la vie est bien connu, il a rarement fait l'objet d'une considération plus attentive, et je me propose donc d'en interroger la signification pour en distinguer trois acceptions en prenant l'exemple de la philosophie diltheyenne de la vie - un exemple dont il convient, pour commencer, de justifier le choix.

${ }^{3}$ Schnädelbach, Philosophie in Deutschland, 1831-1933, op. cit., p. 174. 
Le choix de l'exemple de Dilthey tient à trois ordres de raisons. Si le premier ouvrage comportant l'expression de " philosophie de la vie » dans son intitulé même est le Lebensphilosophie de Friedrich Schlegel de 1828, Dilthey a explicitement assumé cet intitulé pour désigner sa pensée jusque, indirectement ou directement, dans le titre même de ses derniers livres. Indépendamment du titre d'un manuscrit de 1892-93 intitulé «Leben und Erkennen » (Vie et connaissance) sur lequel il nous faudra revenir plus loin, le titre de l'ouvrage Théorie des conceptions $d u$ monde de 1911 porte indirectement sur la vie pour autant que le monde n'est jamais que l'expression du déploiement de la vie, que le monde "nous est donné comme vie ${ }^{4}$. Ainsi, dans le corps de l'ouvrage les termes de vie et de monde semblent pratiquement valoir comme des synonymes, Dilthey écrivant par exemple aussi bien Weltanschauung ou Weltansicht que Lebensansicht, et Weltund Lebensrätsel ${ }^{5}$. De leur côté les deux volumes du Monde de l'esprit parus la même année portent comme sous-titre «Introduction à la philosophie de la vie», Dilthey précisant dans le regard rétrospectif de la préface du Monde de l'esprit que " la tendance prépondérante de [sa] pensée philosophique » a été de « comprendre la vie ${ }^{6}$. Le second ordre de raisons est historique: il tient non seulement au fait que Dilthey a donné sa frappe conceptuelle à la notion d'Erlebnis ou de vécu, mais encore à l'impact de Das Erlebnis und die Dichtung et plus encore au rôle déterminant que sa pensée a pu jouer dans l'élaboration aussi bien de la phénoménologie entre 1905 et 1925 que de l'anthropologie philosophique de Plessner. Le troisième et dernier ordre de raison tient à la postérité plus lointaine si ce n'est à l'actualité de la pensée diltheyenne de la vie. Si les philosophies de la vie ont connu une éclipse, dans la mesure où leur héritage s'est vu assumé par les philosophies de l'existence, Schnädelbach les voit renaître dans les mouvements écologiques - il faudrait surtout nommer Hans Jonas - et altermondialistes, comme dans la pensée de Paul Feyerabend ; comme on le verra, Plessner voit par ailleurs s'esquisser dans la pensée diltheyenne la nouvelle tâche qui s'impose aujourd'hui à la pensée à l'ère de la mondialisation. Qu'en est-il donc de la philosophie diltheyenne de la vie?

On a souvent eu tendance à réduire le projet de Dilthey à la dimension gnoséologique voire épistémologique, c'est-à-dire finalement néo-

\footnotetext{
${ }^{4}$ Dilthey, Gesammelte Schriften - désormais cité GS suivi du tome et de la page -, Stuttgart Göttingen, B. G. Teubner - Vandenhoeck \& Ruprecht, 1990, tome I, p. XIX, trad. S. Mesure, in CEuvres 1, Critique de la raison historique, Paris, Cerf, 1992, p. 149.

${ }^{5}$ Dilthey, GS VIII, respectivement p. 17, 25 et 220, 224.

${ }^{6}$ Dilthey, GS V, 3, trad. CEuvres 1, op. cit., p. 39.
} 
kantienne, de son œuvre en tant qu'elle vise à penser la spécificité des sciences humaines et sociales par rapport à celles dites de la nature ; mais cette manière de réduire le caractère polémique de sa pensée est déjà contredit par le célèbre passage de l'Introduction aux sciences de l'esprit de 1884, selon lequel «dans les veines du sujet connaissant tel que Locke, Hume et Kant le construisirent, ce n'est pas du sang véritable qui coule, mais une sève délayée de raison $»^{7}$. Comment entendre la volonté de « comprendre la vie» ? La préface du Monde de l'esprit en explicite la signification d'abord en précisant vouloir « comprendre la vie à partir d'elle-même ", puis, quelques lignes plus bas après avoir réitéré la nature du projet diltheyen, c'est-à-dire la tâche à laquelle Dilthey a consacré «toute [sa] vie »: "Je désirais pénétrer toujours plus profondément dans le monde historique $»^{8}$.

Autrement dit, la vie, c'est ce qui se donne à nous dans le déploiement de mondes historiques concrets en lesquels elle s'exprime, mais aussi de formes naturelles et de vivants en lesquelles elle s'extériorise, Dilthey ayant toujours insisté sur l'arrière-plan naturel de la vie historique, celui, par exemple de la vie pulsionnelle, ou sur l'entrelacement du naturel et de l'historique. Ces deux dimensions sont deux « aspects » (Aspekten) de la vie ${ }^{9}$ - Dilthey récusant ici toute forme de dualisme métaphysique. La connaissance de la vie implique donc une herméneutique des configurations relevant aussi bien d'expressions culturelles que de "simples » extériorisations naturelles - les Idées pour une psychologie analytique et descriptive invitant à une investigation de la vie psychique aussi bien humaine qu'animale. La critique de la prétention de saisir cette vie à l'aide des concepts d'entendement ne signifie pas pour autant l'inaptitude pour la pensée à appréhender la vie, puisque la pensée appartient elle-même à la vie : elle en a émergé, et elle en est une «fonction $»^{10}$. Et de même que Bergson invitera à appréhender la vie à l'aide de concepts fluides, Dilthey invite à recourir à des «catégories de la vie ${ }^{11}$. Mais cela signifie en second lieu que la connaissance qu'il nous est possible d'avoir de la vie, c'est la connaissance que la vie acquiert d'ellemême. Dire qu'il nous faut comprendre la vie « à partir d'elle-même »

\footnotetext{
${ }^{7}$ Dilthey, GS I, XVIII, trad. Introduction aux sciences de l'esprit, in CEuvres 1, op. cit., p. 148 sq.

${ }^{8}$ Dilthey, GS V, 4, trad. M. Remy, Le monde de l'esprit 1, Paris, Aubier Montaigne, 1947, p. 10.

${ }^{9}$ Dilthey, respectivement GS VII, 79, trad. S. Mesure, L'Édification du monde historique, in CEuvres 7, op. cit., p. 31.

${ }^{10}$ Dilthey, GS V, 223, trad. Le monde de l'esprit I, op. cit., p. 228, et GS XIX, 345 et 318.

${ }^{11}$ Nous nous permettons de renvoyer sur ce point à La pensée herméneutique de Dilthey. Entre néokantisme et phénoménologie, Villeneuve d'Ascq, Presses Universitaires du Septentrion, 2002, p. 150 sq.
} 
ou comme le fait encore "L'essence de la philosophie », que « la vie doit être interprétée (deuten) à partir d'elle-même ${ }^{12}$, c'est dire que "c'est la vie qui saisit la vie ${ }^{13}$, qui «prend conscience d'ellemême ${ }^{14}$. Cette dernière formulation de L'Édification du monde historique, également publiée en 1911 retrouve finalement un thème auquel renvoyait déjà le discours inaugural prononcé en 1867 à l'Université de Bâle, celui, goethéen, d'une nature qui se déploie ou $\mathrm{s}^{\prime}$ explique « afin de jouir d'elle-même » ${ }^{15}$. Autrement dit, l'expression de philosophie de la vie doit être entendue aux deux sens du génitif.

Mais, au regard de ce que montre l'analyse historique, Dilthey récuse la possibilité de trouver pour notre connaissance de la vie un fondement assuré ou certain à partir duquel une déduction serait possible, et à la circularité de la connaissance à laquelle parvient la vie en se déployant, répond en quelque manière la nécessaire circularité de la connaissance humaine, et, en l'occurrence, sa finitude. Si le manuscrit « Leben und Erkennen » déclare ainsi :

L'expression vie dit ce qui nous est à tous le plus connu, le plus intime, mais simultanément le plus sombre, quelque chose de totalement insondable,

cette obscurité est d'abord impliquée par l'historicité de l'être même de l'homme $^{16}$, et donc par le caractère nécessairement préjudiciel de sa connaissance. Dans une perspective qui était déjà celle de Droysen, un des additifs à l'Introduction aux sciences de l'esprit note :

Cercle de la connaissance. Il n'existe pas de point de départ légitime, pas de jugement d'expérience fondé, tout point de départ est au contraire arbitraire. Les différents points de départ des théories de la connaissance sont toujours marqués par l'arbitraire, reposent toujours sur des présupposés ${ }^{17}$.

Ou encore, «il n'existe pas de philosophie sans présupposés » ${ }^{18}$. Dilthey semble ainsi s'inscrire dans une tradition sceptique à laquelle

\footnotetext{
${ }_{12}$ Dilthey, GS V, 370, trad. Le monde de l'esprit I, op. cit., p. 371.

${ }^{13}$ Dilthey, GS VII, 137, trad. L'Édification du monde historique, in CEuvres 7, op. cit., p. 90.

${ }^{14}$ Dilthey, GS VIII, 17, trad. Théorie des conceptions du monde, in CEuvres 7, op. cit., p. 20.

${ }_{15}^{15}$ Dilthey, GS V, 23, trad. Le monde de l'esprit I, op. cit., p. 29.

${ }^{16}$ Dilthey, GS VII, 151, trad. L'Édification du monde historique, in CEuvres 7, op. cit., p. 105.

${ }_{17}$ Dilthey, GS I, 419, trad. L. Sauzin, Introduction aux sciences humaines, Paris, Presses Universitaires de France, 1942, p. 513 sq. En ce qui concerne Droysen, voir Historik, P. Leyh éd., Stuttgart/Bad Cannstadt, Frommann - Holzboog, 1977, p. 7, 418, par exemple.

${ }^{18}$ Dilthey, GS XXIV, 68, et, dans le même sens, dans un manuscrit antérieur à 1880, GS XIX, 339 sq., et GS VII, 137, trad. L'Édification du monde historique, in CEuvres 7, op. cit., 92, GS VIII, 214 - la traduction de la Théorie des conceptions du monde rendant Voraussetzungen par postulats, op. cit., p. 261.
} 
appartiennent Montaigne et Pascal et pour laquelle « la coutume fait nos preuves les plus fortes et les plus crues : elle incline l'automate, qui entraîne l'esprit sans qu'il y pense » - une tradition à laquelle se réfère encore Bourdieu en renvoyant dans ses Méditations pascaliennes au « constat (pascalien) » de «l'arbitraire originel ${ }^{19}$. Ces pré-jugés, au sens étymologique du terme, ne se réduisent pourtant pas à ceux d'une subjectivité qui s'inscrit toujours dans une société et une époque historique données - un thème sur lequel Gadamer mettra comme on sait plus tard encore $l^{\prime}$ accent ${ }^{20}-$, mais relèvent d'orientations ou de comportements affectifs pré-théoriques plus originaires dont la Théorie des conceptions du monde esquisse une généalogie sur laquelle il nous faudra revenir plus loin. Ce caractère préjudiciel et circulaire de la connaissance constitue la première dimension, gnoséologique, du caractère opaque ou insondable de la vie telle qu'il nous est possible de l'appréhender.

À la différence de Pascal néanmoins et au premier abord étrangement, cet insondable n'implique pas pour Dilthey de renoncer à l'exigence de validité universelle de la connaissance à laquelle il a toujours tenu, mais il définit au contraire une tâche infinie, un « but » qu'il « faut se donner de façon toujours plus consciente et critique $»^{21}$. Autrement dit, cette exigence relève d'une Idée au sens kantien de ce terme. Et c'est à cette tâche que se livre ce que l'Introduction aux sciences de l'esprit appelle la "phénoménologie de la métaphysique »: achever la dissolution (Auflösung), "l'euthanasie », c'est-à-dire la Selbstzersetzung de la conceptualité et des systèmes de la métaphysique afin de réaccéder aux expériences originaires qui s'y sont pétrifiées. Le premier des deux versants de cette phénoménologie correspond à la tâche critique que lui assignait pour ainsi dire déjà Lambert ou Kant, alors que le second versant correspond, lui, à la tâche d'une description des vécus. Cette dimension de l'insondabilité de la vie qui tient au caractère indirect, préjudiciel et circulaire de la connaissance qu'il nous est possible d'en avoir reste néanmoins d'ordre théorique, et elle demande à être distinguée d'une dimension plus essentielle qui, elle, est d'ordre pratique si ce n'est existentiel : c'est l'insondable

\footnotetext{
${ }_{19}$ Voir respectivement Pascal, Pensées et opuscules, éd. Brunschvicg, Paris, Hachette, 1912, 252, et Bourdieu, Méditations pascaliennes, Paris, Seuil, « Points », 2003, p. 153.

${ }^{20}$ Gadamer, Wahrheit und Methode, in Gesammelte Werke, tome 1, p. 228 sqq., trad. P. Fruchon et al., Vérité et méthode, Paris, Seuil, 1996, et " Die Universalität des hermeneutischen Problems », in GW 2, p. 224, trad. M. Simon, in L'art de comprendre I, Paris, Aubier Montaigne, 1982, p. 32 (« Ce ne sont pas tant nos jugements que nos préjugés qui constituent notre être »).

${ }^{21}$ Dilthey, GS VII, 137, trad. L'Édification du monde historique, in CEuvres 7, op. cit., p. 92.
} 
de la vie pour un l'homme qui, en tant que vivant, est avant tout incarné.

Il faut, pour le voir, revenir à la manière dont la vie nous est donnée. Elle nous est certes donnée dans une profusion de formes, pour le dire avec Simmel, de formes ou de configurations aussi bien naturelles que culturelles, mais dans le cadre de ce qui s'éprouve, c'est-à-dire d'expériences fondamentales qui sont constitutives de l'existence. Après avoir rappelé que la vie est ce qui nous est le plus intime et simultanément le plus sombre, Dilthey ajoute en effet que cet insondable est celui d'où nous venons et vers lequel nous allons, c'est-à-dire la mort ${ }^{22}$, vers laquelle font aussi signe les «facticités » de la vie: la corruptibilité de toute chose, la cruauté des combats impliqués par la vie, l'évanescence des empires historiques... La Théorie des conceptions $d u$ monde y revient encore vingt ans plus tard en voyant dans la mort «le point central de toutes les incompréhensibilités ${ }^{23}$. C'est sur cet arrière-plan que se déploie ce que Dilthey appelle le chemin de la facticité à l'idéal, ou, en termes husserliens, aux idéalisations de la culture.

L'insondable de la vie, son obscurité, est en ce sens irréductible à celle de préjugés qui, toujours, interdisent d'en avoir une connaissance transparente, ou au caractère indéfini de son flux et de ses extériorisations, à l'apeiron de la "vaste mer », à la " mer infinie » ou encore à la "mer insondable » de ces formes et configurations historiques $^{24}$. L'obscurité de la vie, c'est son caractère sinistre dont Simmel rend compte dans le cadre de ce qu'il appelle la transcendance de la vie. Simmel pointe la contradiction interne à la vie en tant qu'elle ne peut se déployer concrètement qu'en des formes déterminées qui l'obligent à s'autolimiter, ce qui oblige, de ce fait, la vie à constamment briser ces formes ${ }^{25}$. C'est aussi la raison pour laquelle Dilthey qualifie la vie non seulement $d$ 'insondable, mais de manière aussi récurrente d'énigmatique (rätselhaft).

Comme y insistent à juste titre aussi bien Plessner que Bollnow, Dilthey pense néanmoins ce caractère insondable de la vie à partir de son caractère créateur : le déploiement de la vie est une « explication (Explikation) qui est simultanément création ${ }^{26}$. En d'autres termes, l'insondable ne doit pas tant être reconduit à un défaut qu'à la posi-

\footnotetext{
${ }^{22}$ Dilthey, GS XIX, 346 et 357.

${ }^{23}$ Dilthey, GS VIII, 80 sq., trad. Théorie des conceptions du monde, op. cit., p. 110 sq.

${ }^{24}$ Dilthey, respectivement GS VII, 252 et GS V, XLII, Dilthey reprenant ici un thème herdérien d'Une autre philosophie de l'histoire, trad. M. Rouché, Paris, Aubier, bilingue, 1943.

${ }^{25}$ Bollnow, Lebensphilosophie, op. cit., p. 34 sq.

${ }^{26}$ Voir Dilthey, GS VII, 232, et Bollnow, Lebensphilosophie, op. cit., p. 40.
} 
tivité de la vie effective, son caractère à la fois inépuisable et imprévisible ou inanticipable. Cette création demande, là encore, à être entendue en ce qui concerne les configurations aussi bien naturelles que culturelles. «Dans la pierre, dans la fleur, écrit Dilthey, [1'artiste] perçoit et aime le travail créateur et silencieux de la vie, son activité paisible et autonome ${ }^{27}$, et il en va de même des configurations culturelles dans la mesure où "la vie historique est créatrice », où les objectivations ou expressions historiques de la vie sont autant de ses créations ${ }^{28}$. Cette dimension créatrice a été particulièrement thématisée par Bollnow ${ }^{29}$, et antérieurement par Plessner, qui en part pour élaborer au cours des années vingt du siècle dernier son anthropologie philosophique et penser l'homme comme homo absconditus.

Rappelant que l'Unergründlichkeit de la vie a pour la première fois été thématisée par Dilthey, c'est-à-dire avant Nietzsche, Plessner met l'accent sur l'éthos impliqué par la reconnaissance de cet insondable : le caractère " inanticipable » de la vie oblige à voir « la vie passée et sa propre vie dans sa puissance créatrice et simultanément dans sa corruptibilité à partir de l'horizon obscur dont elle provient et vers lequel elle va ${ }^{30}$. C'est dans ce cadre que Plessner invite à penser l'homme comme "puissance » créatrice, et à réentendre ainsi de manière essentiellement pratique la thèse souvent réitérée par Dilthey selon laquelle l'homme ne se connaît pas lui-même par introspection ou en ruminant sur lui-même, mais par l'histoire. Cela signifie, précise Plessner, que l'homme n'est pas seulement conditionné par l'histoire, mais qu'il la conditionne ${ }^{31}$. Plessner en vient ainsi à penser l'homme "théoriquement et pratiquement comme question ouverte", c'est-àdire dans la dimension intermédiaire d'un "entre »: "entre la zone tranquille des repères et configurations signifiantes familières, qui ont toujours déjà été compris» et "l'inquiétante effectivité du monde dénué de sol $»^{32}$. Mais au-delà de l'insondabilité de la vie qui tient au caractère préjudiciel et circulaire de la connaissance qu'il nous est possible d'en avoir, l'insondabilité qui tient à l'inépuisable et à l'inanticipable de la créativité de la vie, cet «entre», fait signe vers une troisième dimension de l'insondable : son caractère abyssal.

\footnotetext{
${ }^{27}$ Dilthey, GS VI, 226 sq., trad. CEuvres 7, op. cit., 158.

${ }^{28}$ Dilthey, respectivement GS VII, 153 et 220.

${ }^{29}$ Voir Bollnow, Lebensphilosophie, op. cit., p. 37-46.

30 Plessner, Macht und menschliche Natur (1931), Gesammelte Schriften, tome V, Francfort-sur-leMain, Suhrkamp, 2003, p. 184.

31 Voir respectivement Dilthey, GS V, 180, trad. Le monde de l'esprit I, op. cit., p. 186, et GS VII, 87, 224, 279, et Plessner, Macht und menschliche Natur, op. cit., p. 190, 191.

32 Plessner, Macht und menschliche Natur, op. cit., p. 188 et 197.
} 
Cette troisième dimension est thématisée de la manière la plus radicale par la Théorie des conceptions du monde dans un célèbre passage intitulé « Rêve ». La Théorie des conceptions du monde insiste à plusieurs reprises sur l'irréductible «anarchie ${ }^{33}$ qui règne parmi la diversité des conceptions poétiques, religieuses et philosophiques du monde, qui luttent pour la suprématie, et que Dilthey reconduit non pas à des thèses purement théoriques comme le fait la dialectique transcendantale de la Critique de la raison pure, mais à des tonalités affectives primitives qui déterminent autant de visions et de comportements ou positions à l'égard de la vie et donc du monde, avant de se voir élaborées conceptuellement et langagièrement en " conceptions ». S'il est inutile de s'arrêter ici à la tentative diltheyenne de procéder à une typologie des conceptions du monde qui dérivent de ces visions, puisqu'il n'accordait lui-même qu'une valeur heuristique et provisoire à cette typologie, il convient en revanche de considérer le «rêve» rapporté par Dilthey. Ce rêve montre en effet non pas simplement une vie qui se différencie en des formes et configurations - ce qui témoigne seulement de l'obscurité de la création -, mais une vie qui diffère d'elle-même, ce qui, en revanche, rend son unité problématique, c'est-à-dire la révèle comme abyssale.

Le récit de ce rêve, dont la description est immédiatement aussi sinistre que celle des facticités de la vie et de l'énigmaticité de la mort, se déploie en trois actes. Le premier acte décrit le contexte du rêve : la contemplation, avant l'endormissement, de la paix et de l'harmonie qui se dégagent de L'école d'Athènes de Raphaël dont une reproduction ornait l'un des murs de la chambre de Dilthey dans la demeure de son ami York von Wartenburg. Le second acte, le rêve, voit les personnages de ce rêve s'animer et rejoints par des philosophes, poètes, historiens, postérieurs à l'époque de Raphael, et qui se regroupent selon leurs affinités - celles que cherchent à circonscrire la typologie diltheyenne des conceptions du monde. Seulement, le regroupement de ces figures implique simultanément l'éloignement de chaque groupe par rapport aux autres jusqu'au point où ils se voient séparés par une étrangèreté " terriblement hostile ", jusqu'à ce que s'effondre le sol qui les portait. Si le statut de ce rêve pourrait ainsi être comparé à la seconde des Méditations métaphysiques de Descartes, sa tonalité est en revanche très différente dans la mesure où le rêveur est confronté non pas à la possibilité de la tromperie, mais à celle d'une dislocation.

${ }^{33}$ Dilthey, GS VIII, 75, 78, 224 et 256, par exemple, trad. Théorie des conceptions du monde, op. cit., p. $93,97,272$ et 307 . 
Contrastant avec la paix et l'harmonie qui se dégageait avant l'endormissement de la contemplation de L'école d'Athènes, une angoisse surgit ainsi au cœur de ce rêve face à la dissolution de la philosophie, qui apparaît alors trois fois " ou peut-être même plus » à Dilthey, une philosophie dont "l'unité de [s]on être semblait se déchirer $»^{34}$. Si l'on comprend bien le refus husserlien de la possibilité $\mathrm{d}^{\prime}$ une telle «terrible anarchie » comme dit encore Dilthey - ce qui est évidemment à entendre littéralement comme absence de tout principe ou de tout arche -, et si ce rêve semble confirmer le diagnostic de Schnädelbach selon lequel l'un des traits des philosophies de la vie, c'est leur tragique, le troisième acte du récit de ce rêve est aussi inattendu que le second par rapport au premier. Dilthey s'éveille en effet de son rêve pour entrer dans une rêverie dont la tonalité est au contraire heureuse : le sentiment dominant de cette rêverie, c'est celui non pas - comme l'ont parfois pensé certains lecteurs peut-être trop pressés - d'une résignation face à l'impossibilité de conquérir un sol assuré pour notre savoir, mais d'une libération. Contemplant l'univers sidéral qui s'offre à ses yeux et dont les astres lui paraissent briller encore plus clairement, "saisi » par son caractère "incommensurable, insaisissable et insondable » qui reflète la multiplicité des figures de son rêve, le "doute absolu » si ce n'est le désespoir qui l'avait étreint se voit en effet dissipé. D'abord, car la conscience historique permet finalement de légitimer chacune des conceptions du monde, c'est-à-dire de guérir la blessure qu'elle s'est elle-même infligé, Dilthey thématisant de manière bien plus ample ce que remarquait déjà un manuscrit de 1874-79: l'unilatéralité de tous les systèmes ${ }^{35}$. Ensuite, car la Théorie des conceptions du monde permettrait de dégager les lois qui régissent le déploiement de leur diversité.

En d'autres termes, cela signifie d'une part que « la pure lumière de la vérité ne peut être aperçue qu'à travers la diversité des rayons en lesquels elle se réfracte », et d'autre part que "l'homme se trouve désormais complètement libre $»^{36}$. Mais il faut encore remarquer la tonalité finale du récit de ce rêve, dont, éveillé, Dilthey essaie en vain de retenir «les images heureuses». Dans la continuité d'un passage antérieur de la Théorie des conceptions du monde qui décrivait le visage de la vie, c'est-à-dire ce qu'elle nous donne à voir, comme «tantôt

\footnotetext{
${ }^{34}$ Dilthey, GS VIII, 223, trad. Théorie des conceptions du monde, op. cit., p. 271.

${ }^{35}$ Dilthey, GS XVIII, 200.

${ }^{36}$ Dilthey, GS VIII, 225, trad. Théorie des conceptions du monde, op. cit., p. 273. Indépendamment de la différence de leurs analyses, Bourdieu qualifie de manière similaire de "par soi salutaire et libérateur » le «constat (pascalien) [...] de l'arbitraire originel » (Méditations pascaliennes, op. cit., p. 153).
} 
souriant, tantôt se contractant de souffrance », « Rêve » poursuit : "Notre génération cherche à lire dans le visage mystérieux et impénétrable de la vie à la bouche souriante et aux yeux si chargés de mélancolie ${ }^{37}$. Loin qu'il s'agisse là d'un passage isolé de son œuvre, celui-ci reprend pratiquement littéralement les derniers mots de "L'esthétique de l'époque moderne et sa tâche actuelle » de 1892 : «Toutes les générations de penseurs et de poètes cherchent à lire dans la face secrète, insondable de la vie, sur ses lèvres rieuses et dans ses yeux au regard mélancolique, et cela non plus n'a pas de fin $»^{38}$. Autrement dit, le savoir de la vie est donc, aussi opaque, fragmentaire et terrible, aussi tragique ou peu tragique qu'il soit, un gai savoir.

La proximité entre Nietzsche et Dilthey est ici patente. Certes, le second ne pouvait suivre le renoncement du premier à l'exigence de validité universelle dans la connaissance ${ }^{39}$, et il n'y a qu'une lointaine analogie entre la comparaison diltheyenne des sciences de l'esprit à un « pont», à un "vaisseau au-dessus de l'insondable océan $»^{40}$, et la comparaison nietzschéenne entre l'homme et un " pont», une « corde tendue au-dessus de l'abîme ", puisque cette comparaison n'a de sens que par rapport à la notion de surhumain. La proximité des deux penseurs est en revanche plus essentielle en ce qui concerne précisément ce qu'il faut entendre par vie. Le «Chant de la danse » du Zarathoustra consonne avec la perception diltheyenne d'une vie au sourire mystérieux lorsque Nietzsche écrit : «Un jour j'ai contemplé tes yeux, oh vie! Et il me semblait tomber dans un abîme insondable. Mais [...] tu avais un rire moqueur quand je te nommais insondable $»^{41}$. Indépendamment du fait qu'il y a sourire et sourire, sourire et rire, si donc l' «énigme de la vie » constitue «l'unique, l'obscur, l'effrayant objet de toute philosophie ${ }^{42}$, il n'en reste pas moins que, comme le rappellent Plessner et Bollnow, qui y voit une caractéristique en général des philosophies de la vie, la tonalité fondamentale de la pensée diltheyenne est affirmative et joyeuse ${ }^{43}$.

\footnotetext{
${ }^{37}$ Dilthey, GS VIII, respectivement 140 et 226, trad. Théorie des conceptions du monde, op. cit., p. 176 et 274 .

${ }_{38}$ Dilthey, GS VI, 287, trad. CEuvres 7, op. cit., p. 222.

${ }^{39}$ Dilthey, GS VIII, 199 sqq., trad. Théorie des conceptions du monde, op. cit., p. 204-206.

${ }^{40}$ Dilthey, GS V, XLII.

${ }^{41}$ Nietzsche, Ainsi parlait Zarathoustra, "se tenir dans l'abîme " à partir d'une lecture de Hans Lipps et de Nietzsche, Kerckhoven, "Le lointain et l'étranger", in Faire monde, M. Foessel, J.-C. Gens et P. Rodrigo éd., Paris, Mimesis, 2011, p. 125-144.

${ }^{42}$ Dilthey, GS VIII, 140, trad. Théorie des conceptions du monde, op. cit., p. 176.

${ }^{43}$ Voir Plessner, Macht und menschliche Natur, op. cit., p. 194, et Bollnow, Lebensphilosophie, op. cit., p. 101 sq.
} 
Le caractère énigmatique et insondable de la vie telle que la pense Dilthey tient donc simultanément à son opacité - elle est donc pour nous non pas un fait ou une évidence, mais une question -, à son caractère créateur, c'est-à-dire inépuisable et inanticipable, et enfin et surtout, à son caractère abyssal dont l'anarchie des conceptions du monde est l'expression. Il est à cet égard difficile $d^{\prime} y$ voir le résidu d'une sécularisation de l'idée théologique de l'insondabilité divine, ou une "notion » confuse sauf à penser que la réalité est elle-même transparente. La singularité d'une telle philosophie de la vie qui récuse, comme celle de Nietzsche, la possibilité d'assigner un centre à la vie en tant qu'elle diffère essentiellement d'elle-même semble évidente par rapport à une philosophie de la vie comme celle de Bergson. Malgré leur commune conception de la vie comme création, il paraît difficile d'imaginer que Dilthey aurait, par exemple, pu signer le passage de L'évolution créatrice dans lequel Bergson écrit à propos de la vie : "Je parle d'un centre d'où les mondes jailliraient comme les fusées d'un immense bouquet [...]. La création ainsi conçue n'est pas un mystère ${ }^{44}$.

La conception diltheyenne de la vie qui pointe l'écart ou la différence de la vie par rapport à elle-même implique par ailleurs d'une part une révision de la notion d'homme et de subjectivité. Car en tant qu'il, ou elle, appartient à cette vie, leur unité ne peut être pensée comme un donné immédiat, et ils demanderaient à être d'abord pensés en termes nietzschéens de chaos. Mais, comme le suggère Plessner, cette philosophie de la vie rend $\mathrm{d}^{\prime}$ autre part aujourd'hui impossible d'en rester à $\mathrm{l}^{\prime}$ " absolutisation » des concepts et des valeurs que l'Occident pensait pouvoir imposer au reste du monde ${ }^{45}$; Dilthey esquisse ainsi aux yeux de Plessner l'attitude philosophique appelée par ce que nous appelons aujourd'hui l'époque de la mondialisation où, comme l'avançait aussi Merleau-Ponty dans «Partout et nulle part», la philosophie est appelée à s'ouvrir à des modalités différentes de la pensée.

\footnotetext{
${ }^{44}$ Bergson, L'évolution créatrice, Paris, Presses Universitaires de France, 1969, p. 249 (je souligne, J.-C. Gens).

45 Plessner, Macht und menschliche Natur, op. cit., p. 185 sq. et 189.
} 